\title{
How the World first saw Atoms: An Outline of how Field Ion Imaging works
}

\author{
Richard G. Forbes \\ University of Surrey, Advanced Technology Institute (BB), \\ School of Electronics and Physical Sciences, Guildford, Surrey GU2 7XH, UK.
}

The world first saw atoms in the autumn of 1955, when Kanwar Bahadur and Erwin Müller invented the low-temperature field-ion microscope (FIM) at Pennsylvania State University. Melmed has described this period [1]. It then took about 30 years for a reasonable consensus to be reached on the qualitative principles of how field-ion imaging happens. The mechanism is complicated, and still beyond detailed quantitative modelling. There has been little development in the last 10 years or so. But, for this "Golden Anniversary of Imaging Atoms", it seems useful to present an outline of our current understanding of the field-ion imaging mechanism. Space limitations make it impracticable to present supporting images and diagrams here; back-up material can be found in Refs [2,3].

The FIM images a pointed emitter of apex radius typically $60 \mathrm{~nm}$, by the selective field ionization of an imaging gas (typically helium or neon) slightly above protruding atoms in the emitter surface. The resulting imaging-gas ions then travel approximately radially to an image-intensification device, which produces a magnified image of the points of highest electric field at the emitter surface.

Most of the ionization occurs in an ionization zone close above the so-called critical surface, which is typically about $4 \mathrm{~nm}$ above the emitter's geometrical surface. When the nucleus of the imaginggas atom is at (or outside) the critical surface, then its electron can tunnel to an emitter electron state at (or above) the emitter Fermi level. When the nucleus is inside the critical surface then tunneling is forbidden. However, local field-induced adsorption of gas atoms takes place inside the forbidden zone. The imaging-gas atoms thus "bounce around" on top of a layer of field-adsorbed gas atoms. The field-adsorbed layer has an important role in cooling and trapping "hot" incoming gas atoms.

Associated with the ionization above a protruding metal atom there is a characteristic ionization density $j_{\mathrm{A}}$ that occurs at a characteristic point " $\mathrm{A}$ " in the critical surface. Point "A" is above the protruding atom, near the centre of its ionization zone. This ionization density $j_{\mathrm{A}}$ is given by

$j_{\mathrm{A}}=1 / 2 C_{\mathrm{A}} P_{\mathrm{eA}}$

where $P_{\mathrm{eA}}$ is the field ionization rate-constant for an atom with its nucleus at point $\mathrm{A}$, and $C_{\mathrm{A}}$ is the gas concentration applicable to point $A$ (i.e. the probability per unit volume of finding a gas-atom nucleus in the vicinity of point A). To discuss contrast in field-ion images, one needs to consider a ratio of ionization densities at two characteristic points "A" and "B". This ratio is given by

$j_{\mathrm{A} / j_{\mathrm{B}}}=\left(C_{\mathrm{A}} / C_{\mathrm{B}}\right)\left(P_{\mathrm{eA}} / P_{\mathrm{eB}}\right)$

Point "B" may either be a characteristic point above another atom (when discussing relative spot brightness), or a characteristic minimum at a point in the critical surface midway between neighbouring surface atoms (when discussing atomic resolution). 
To determine $\left(C_{\mathrm{A}} / C_{\mathrm{B}}\right)$ one has to determine the kinetic history of the imaging gas distribution, and the point in the history at which the gas gets ionized. This is very complicated [2]. A typical gasatom history involves: (1) The supply and capture of imaging gas, mainly in the high-field region stretching from the "cap"' down the emitter "shank". (2) Attraction of the trapped gas to the imaged emitter cap, under the influence of polarization forces, with "heating" of the gas during this process. (3) Cooling down of the gas as it bounces on the field-adsorbed gas layer, and its "capture" into regions of the surface, which is influenced by the complicated field-induced polarization-potential structure at the surface. (4) "Diffusion" of the cooled gas across the emitter surface, in order to build up the field-induced gas-concentration variations that are characteristic of a Maxwell-Boltzmann equilibrium. Field ionization typically occurs towards the end of stage 3.

A result is a set of statements that apply to best imaging conditions. (1) The relative brightnesses of different general areas of a field-ion image are determined by gas-supply considerations. (2) The relative brightnesses of adjacent spots, and atomic resolution issues, are determined by local-gasequilibrium considerations. (3) Spot size is influenced by the lateral gas velocity at ionization. (4) When field ionized the gas is slightly hot, i.e. above the emitter temperature. (5) At emitter temperatures near $80 \mathrm{~K}$ the rate-constant ratio $\left(P_{\mathrm{eA}} / P_{\mathrm{eB}}\right)$ is the dominant factor in contrast formation, but at much lower temperatures (near $5 \mathrm{~K}$ ) the gas-concentration ratio $\left(C_{\mathrm{A}} / C_{\mathrm{B}}\right)$ is dominant.

For the rate-constant $P_{\mathrm{e}}$, an approximate numerical theory is as follows. For an atom in free space:

$P_{\mathrm{e}}=v_{\mathrm{e}} \exp \left\{-\mu b I^{3 / 2} / f\right\}$

where: $f$ is mean electric field in the tunnelling barrier (typically around $45 \mathrm{~V} / \mathrm{nm}$ for He imaging); $I$ is the atom's first ionization energy $(24.6 \mathrm{eV}$ for $\mathrm{He}) ; b$ is the second Fowler-Nordheim constant $\left(\cong 6.83 \mathrm{eV}^{-3 / 2} \mathrm{~V} \mathrm{~nm}^{-1}\right) ; \mu$ is a correction factor of order unity; $v_{\mathrm{e}}$ is an "effective frequency of electron escape attempts" (typically $10^{16} \mathrm{~Hz}$ ). What matters is the sensitivity of $P_{\mathrm{e}}$ to field variations. Let the rate-constant increase from $P_{\mathrm{e} 0}$ to $P_{\mathrm{e}}$ when the field increases from $f_{0}$ to $f=f_{0}+\delta f$ : we define a total field sensitivity $s_{f}$ by $P_{\mathrm{e}} / P_{\mathrm{e} 0}=\exp \left\{s_{f}\left(\delta f / f_{0}\right)\right\}$. For free space it can be shown that $s_{f} \cong 17$.

Close above the surface, the theory is more complex, and $s_{f}$ is lower, perhaps around 5 . A $10 \%$ field variation then yields a rate-constant ratio of about 1.6; this implies an ionization-density ratio of at least 1.6. It turns out that $f$ should be identified as the average electric field between the critical surface and the emitter's electrical surface. So, if the variation in $f$ across a surface, between a point between atoms and a point above an atom, is close to $10 \%$ or more, then the Raleigh criterion allows neighbouring atoms to be resolved. Modelling suggests this condition is satisfied for adjacent atoms on crystal facets such as tungsten (111), which is indeed resolved by FIM. So this is how we "first saw atoms": at a strongly charged surface, the electric field associated with the protons in an atomic nucleus "shines through" the surrounding electrons: so the electric field is higher over atoms than between them: so field ionization creates more ions above atoms than between them: in the FIM these ions form a projection image, and "atomic spots" appear if the field variations are large enough.

\section{References}

[1] A.J. Melmed, Appl. Surface Sci. 94/95 (1996) 17.

[2] R.G. Forbes, Appl. Surface Sci. 94/95 (1996) 1.

[3] R.G. Forbes, J. Phys D: Appl. Phys. 18 (1985) 973. 